\title{
Development of an UPLC-MS/MS Method for the Quantitative Analysis of Upadacitinib in Beagle Dog Plasma and Pharmacokinetics Study
}

\author{
Meng-Jie Wang' \\ Yu-Hang Zhao $\mathbb{D D}^{2}$ \\ Chen Fan (1D $)^{3}$ \\ Ying-Jie Wang $\mathbb{D}^{3}$ \\ Xin-Qi Wang $\mathbb{D}^{3}$ \\ Xiang-Jun Qiu (iD ${ }^{3}$ \\ Rui-Le Shen'
}

'Department of Neurology, The First Affiliated Hospital, and College of Clinical Medicine of Henan University of Science and Technology, Luoyang, Henan, 47I003, People's Republic of China; ${ }^{2}$ School of Medical Imaging, Hangzhou Medical College, Hangzhou, 31005I, Zhejiang, People's Republic of China; ${ }^{3}$ Department of Pharmacy, School of Basic Medical Sciences, Henan University of Science and Technology, Luoyang, 471023, Henan, People's Republic of China
Correspondence: Xiang-Jun Qiu Department of Pharmacy, School of Basic Medical Sciences, Henan University of Science and Technology, Luoyang, 471023, Henan, People's Republic of China Email lyxiangjun@I26.com

Rui-Le Shen

Department of Neurology, The First Affiliated Hospital, and College of Clinical Medicine of Henan University of Science and Technology, Luoyang, 47I003, Henan, People's Republic of China

Email yfyshenruile@I26.com
Background: Upadacitinib, a novel selective Janus kinase 1 (JAK1) inhibitor, has been recently approved by the US FDA for the treatment of adult patients with moderately to severely active rheumatoid arthritis (RA). An ultra-performance liquid chromatography tandem mass spectrometry (UPLC-MS/MS) method for the quantitative analysis of upadacitinib in beagle dog plasma was developed and validated.

Methods: Upadacitinib and fedratinib (internal standard, IS) were extracted with ethyl acetate under alkaline condition and then separated and detected. The chromatographic column was Waters Acquity UPLC BEH C18 column $(2.1 \mathrm{~mm} \times 50 \mathrm{~mm}, 1.7 \mu \mathrm{m})$, the mobile phase was acetonitrile and $0.1 \%$ formic acid in water with gradient elution procedure, and the flow rate was $0.40 \mathrm{~mL} / \mathrm{min}$. Under the positive ion mode, upadacitinib and IS were monitored by multiple reaction monitoring (MRM) as the following mass transition pairs: $\mathrm{m} /$ $z 447.00 \rightarrow 361.94$ for upadacitinib and $m / z 529.82 \rightarrow 141.01$ for IS.

Results: In the concentration range of $1-500 \mathrm{ng} / \mathrm{mL}$, upadacitinib had good linearity, and the lower limit of quantification (LLOQ) was $1 \mathrm{ng} / \mathrm{mL}$. The RSD of the intra- and inter-day precision was less than $10.03 \%$, and the RE of accuracy was $-3.79 \%$ to $2.58 \%$. The extraction recovery of upadacitinib was more than $80 \%$, the matrix effect was around $100 \%$, and upadacitinib was found to be stable.

Conclusion: The novel optimized UPLC-MS/MS assay was an effective tool for the determination of upadacitinib and had been successfully applied to the pharmacokinetic study of upadacitinib in beagle dogs, and this method would also be used to study DDIs.

Keywords: upadacitinib, UPLC-MS/MS, pharmacokinetic, beagle dog

\section{Introduction}

Rheumatoid arthritis (RA) is a chronic systemic inflammatory autoimmune disease, which can lead to severe pain, disability and progressive joint destruction. Without adequate treatment, irreversible joint destruction and loss of function can lead to severe disability and impaired quality of life. ${ }^{1,2}$ In recent 30 years, with the development of biological treatment methods such as anti TNF, anti IL-6, CTLA4 Ig and anti CD20, the treatment of RA has undergone revolutionary changes. The effectiveness of these treatments provides patients with a variety of treatment methods. ${ }^{3}$ Based on this clinical understanding, drugs targeting a variety of pro-inflammatory mechanisms, such as the Janus kinase (JAK) family of intracellular signaling enzymes, are being developed for the treatment of RA. ${ }^{3}$ 
JAK enzymes are tyrosine kinases that can bind to the cytoplasmic region of cytokine receptors and are activated after the cytokines bind to their cellular receptors. The JAK family (JAK 1, 2, 3 and tyrosine kinase 2 [TYK 2]) are important mediators of a variety of cytokine signaling pathways involved in normal cellular processes, and also important mediators in the pathogenesis of RA and other immune-mediated inflammatory diseases. ${ }^{2,4}$

Oral JAK inhibitors belong to the class of targeted synthetic disease-modifying antirheumatic drugs (DMARD). At present, they are recommended as a treatment option for RA patients worldwide. Although these patients have received traditional synthetic DMARD (csdmard) treatment, they still have moderate or high disease activity, or they fail to treat with biological drug DMARD (bdmard). ${ }^{5,6}$ It has been hypothesized that selectively inhibiting JAK1 block intercepts pathogenic cytokine signaling in RA while retaining other signaling pathways required for normal physiological functions. ${ }^{7}$

Upadacitinib (Figure 1A), a novel selective JAK1 inhibitor, was recently approved by the US FDA for the treatment of adult patients with moderately to severely active RA. ${ }^{8}$ Upadacitinib $15 \mathrm{mg}$ demonstrated a favorable benefitrisk profile and filled an unmet medical need for patients with RA, providing significantly greater rates of disease outcomes (remission and LDA) compared with established therapies (methotrexate, adalimumab, and abatacept), and inhibits structural joint damage. ${ }^{9}$ Upadacitinib could be a favorable long-term treatment option in patients with PsA who are refractory to biologic therapy. ${ }^{10}$

Upadacitinib is mainly metabolized by CYP3A and a small part by CYP2D6 in vitro. ${ }^{11}$ The AUC and $\mathrm{C}_{\max }$ of Upadacitinib are significantly affected by strong CYP3A4

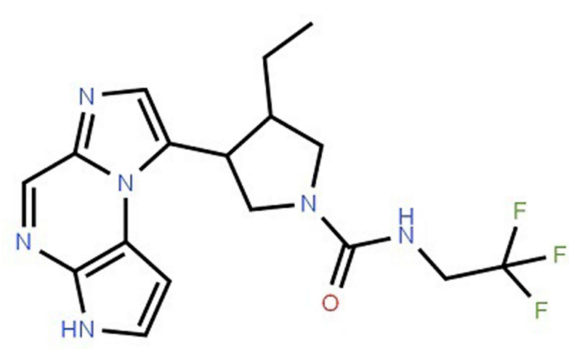

A inhibitors and strong CYP3A4 inducers. Therefore, in patients receiving long-term treatment with strong CYP3A4 inhibitors, it is recommended to use upadacitinib carefully, and it is not recommended to use upadacitinib with strong CYP3A4 inducer. ${ }^{12}$ Therefore, it is necessary to develop a quantitative analysis method of upadacitinib to study the pharmacokinetics and drug-drug interactions (DDI).

UPLC-MS/MS was a rapid, sensitive and accurate method for the determination of drug concentration in biological samples. ${ }^{13,14}$ There have been reports on the human pharmacokinetics of upadacitinib, but the description of the detection of plasma upadacitinib concentration is incomplete. ${ }^{11,15,16}$ At present, there is no literature on the complete methodological validation of the detection of plasma upadacitinib concentration. Therefore, in this experiment, we developed and validated a sensitive and quick UPLC-MS/MS assay for the determination of upadacitinib in beagle dog plasma, and fedratinib was used as the internal standard (IS, Figure 1B). And, the novel developed and validated UPLC-MS/MS method was successfully employed to study the pharmacokinetics of upadacitinib in beagle dogs.

\section{Materials and Methods}

\section{Chemicals Materials}

The purity of upadacitinib and fedratinib were $>98 \%$ and were purchased from Beijing sunflower technology development CO., LTD (Beijing, China). Methanol and acetonitrile in this study were HPLC grade and were purchased from Tianjin Kermel Chemical Reagent Co., Ltd (Tianjin, China). Analytically pure formic acid was purchased from Tianjin Kermel Chemical Reagent Co., Ltd (Tianjin, China).

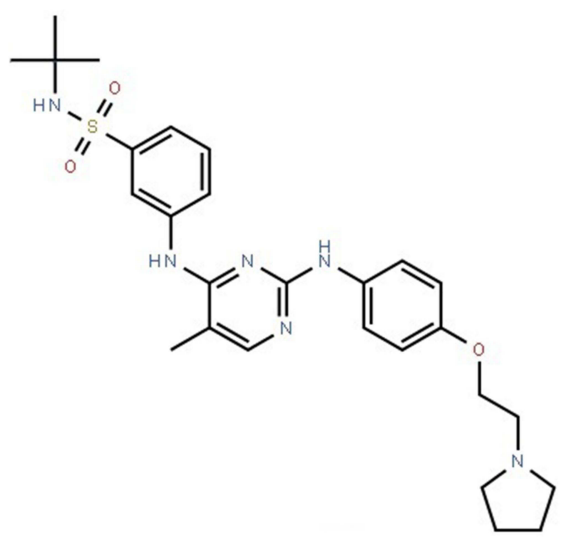

B

Figure I The chemical structure upadacitinib (A) and fedratinib (IS, B). 


\section{Instruments}

Waters ACQUITY UPLC I-Class instrument equipped with online degasser, automatic sampler, four element pump (Waters, USA). Waters XEVO TQD triple quadrupole mass spectrometer equipped with electrospray ionization (ESI) source (Waters, USA). Other instruments included electronic analytical balance and vortex mixer, ultra-pure water equipment, etc.

\section{Solutions Preparation}

Accurately weigh $10 \mathrm{mg}$ of upadacitinib in a volumetric flask, dissolve it with methanol and fix the volume to obtain $1 \mathrm{mg} / \mathrm{mL}$ standard stock solution. $1 \mathrm{mg} / \mathrm{mL}$ IS stock solution was prepared using the same method. The stock solution of $1 \mathrm{mg} / \mathrm{mL}$ upadacitinib was diluted 10 times with methanol to obtain the standard solution of 100 $\mu \mathrm{g} / \mathrm{mL}, 10 \mu \mathrm{g} / \mathrm{mL}$ and $1 \mu \mathrm{g} / \mathrm{mL}$ for calibration curve and quality control (QC) samples.

Blank beagle dog plasma was used to dilute the standard solution and prepare different concentrations of plasma standard solution. The calibration curves with eight different concentrations of upadacitinib were prepared, and the concentration of calibration curve in plasma was as follows: $1,2.5,5,10,25,50,100,200 \mathrm{ng} / \mathrm{mL}$. QC samples of low, medium and high concentrations $(2.5,50$ and $150 \mathrm{ng} / \mathrm{mL}$ ) were prepared in the same way. The IS working solution at a concentration of $100 \mathrm{ng} / \mathrm{mL}$ was obtained by dilution of its stock solution with methanol.

\section{Plasma Sample Treatment}

$100 \mu \mathrm{L}$ of beagle dog plasma to be tested was taken into a $1.5 \mathrm{~mL}$ EP tube, $20 \mu \mathrm{L}$ of internal standard working solution was added, and mixed well. $200 \mu \mathrm{L}$ of $1 \mathrm{~mol} / \mathrm{L}$ sodium hydroxide $(\mathrm{NaOH})$ solution was added and mixed well. Then, $1 \mathrm{~mL}$ ethyl acetate was added and vortexed for $1 \mathrm{~min}$. Took the upper organic phase into another $1.5 \mathrm{~mL}$ EP tube and blew dry with nitrogen flow. The residue was dissolved with $100 \mu \mathrm{L}$ mobile phase, take $50 \mu \mathrm{L}$ into the sample of an automatic injector, and $5 \mu \mathrm{L}$ supernatant was injected into UPLC-MS/MS for detection.

\section{Analytical Conditions}

The chromatographic column was Waters Acquity UPLC BEH C18 column $(2.1 \mathrm{~mm} \times 50 \mathrm{~mm}, 1.7 \mu \mathrm{m})$, and the mobile phase was $0.1 \%$ formic acid in water (A) and acetonitrile (B) with gradient elution procedure, the flow rate was $0.40 \mathrm{~mL} / \mathrm{min}$. The gradient elution procedure was as follows: $0-0.5 \min \mathrm{A} 90 \rightarrow 10 \%, 0.5-1.0 \mathrm{~min} \mathrm{~A} 10 \%$, $1.0-1.1 \mathrm{~min} \mathrm{~A} \quad 10 \rightarrow 90 \%$, and $1.1-2.0 \mathrm{~min} \mathrm{~A} 90 \%$. The column was maintained at $40^{\circ} \mathrm{C}$, the temperature of the autosampler tray was set at $4^{\circ} \mathrm{C}$. The volume of each injection was $5.0 \mu \mathrm{L}$.

Under the positive ion mode, upadacitinib and IS were monitored by multiple reaction monitoring (MRM) as the following mass transition pairs: $\mathrm{m} / z \quad 380.95 \rightarrow 255.97$ for fedratinib and $\mathrm{m} / \mathrm{z} 525.12 \rightarrow 98.00$ for IS. The collision energy and cone voltage of upadacitinib and IS were 25 $\mathrm{V}$ and $30 \mathrm{~V}$, respectively. The desolvation temperature was $1000^{\circ} \mathrm{C}$, the capillary voltage was $2.0 \mathrm{kV}$. Masslynx 4.1 (waters, USA) was used for data acquisition and instrument control.

\section{Method Validation}

Methodology validation of UPLC-MS/MS included specificity, standard curve, LLOQ, precision, recovery, matrix effect and stability. According to the principles of Industry Bioanalytical Method Validation proposed by FDA, and the technical guidelines for nonclinical pharmacokinetics of chemical drugs (China Food and Drug Administration, CFDA), the UPLC-MS/MS method was validated. ${ }^{17,18}$

By comparing the chromatograms of blank beagle dog plasma samples from different sources, beagle dog blank plasma spiked with upadacitinib and IS, and beagle dog plasma sample after administration of upadacitinib, the specificity of UPLC-MS/MS method was evaluated.

The plasma standard solution with the concentration of $1,2.5,5,10,25,50,100,200 \mathrm{ng} / \mathrm{mL}$ of upadacitinib was prepared and detected according to the plasma sample preparation method. The peak areas of upadacitinib and IS were recorded, respectively. The ratio of the peak area of upadacitinib to the IS peak area was taken as the ordinate $y$, the concentration of corresponding points was taken as the abscissa $\mathrm{x}$, and the standard curve of upadacitinib was drawn with the least square method with a weighted $\left(1 / x^{2}\right)$. The lowest concentration of the standard curve was the LLOQ.

Plasma quality control samples of low, medium and high concentrations levels $(2.5,50$ and $150 \mathrm{ng} / \mathrm{mL})$ were taken and injected according to the item of "plasma sample treatment". Six samples of each concentration were measured in parallel and detected within one day to calculate the intra-day precision and accuracy. The same method was used for 3 consecutive days to calculate the inter-day precision and accuracy. The precision was 
expressed by relative standard deviation (RSD, \%) and the accuracy was expressed by relative error (RE, \%).

At low, medium and high concentration levels $(2.5,50$ and $150 \mathrm{ng} / \mathrm{mL}$ ), the extraction recovery was determined by comparing the ratio of the peak area of upadacitinib before and after the extraction, respectively. Matrix effect (ME) was also analyzed in 6 replicates by comparing the response of upadacitinib in plasma matrix after extraction with that in neat solution.

At low, medium and high concentration levels (2.5, 50 and $150 \mathrm{ng} / \mathrm{mL}$ ), the stability of plasma samples was investigated under four different storage conditions: room temperature for $4 \mathrm{~h}$, processed samples at $4^{\circ} \mathrm{C}$ in autosampler tray for $6 \mathrm{~h}$, three freeze-thaw cycles $\left(-20^{\circ} \mathrm{C}\right.$ to $\left.25^{\circ} \mathrm{C}\right),-20^{\circ} \mathrm{C}$ for 4 weeks.

\section{Animal Experiments}

Six beagle dogs (weight $7.5 \sim 9.5 \mathrm{~kg}$ ) were purchased from Hubei yizhicheng Biotechnology Co., Ltd (Shiyan, HUBEI), and the animal license number was SCXK (HUBEI) 20160020. The six beagle dogs were raised in the Laboratory Animal Center of Henan University of Science and Technology (Luoyang, China). All the experimental behaviors and operations were approved by the Institutional Ethics Committee of Henan University of Science and Technology (Luoyang, China), and the ethical approval number of the animal experiment was 202012003. The experimental operation was carried out according to the rules for the Care and Use of Laboratory Animals.
All the beagle dogs were free to access the water and had a $12 \mathrm{~h}$ fasting before the experiment. Upadacitinib was dissolved in $0.5 \%$ carboxymethyl cellulose sodium (CMC-Na) solution and was orally administered to beagle dogs at a dose of $1 \mathrm{mg} / \mathrm{kg}$. At the different time points of $0,0.33,0.67,1,1.5,2$, $3,4,6,9,12,24$, and $48 \mathrm{~h}$, approximate $1.0 \mathrm{~mL}$ blood samples were collected from the veins of the anterior and posterior limbs and taken into $1.5 \mathrm{~mL}$ heparinized EP tubes. The plasmas were separated by centrifugation for 10 minutes at $10,000 \mathrm{rpm}$, and the plasmas were collected and kept frozen at $-20^{\circ} \mathrm{C}$.

\section{Plasma Sample Detection}

Using batch processing method, the concentration of upadacitinib in beagle dog plasma was detected by the developed UPLC-MS/MS technique in this study. The analytical batch included standard curve and QC samples.

\section{Statistical Analysis}

DAS (Drug and Statistics, version 2.0) was used to calculate the important pharmacokinetic parameters of upadacitinib through statistical moment method. The main pharmacokinetic parameters of upadacitinib were as follows: $\mathrm{T}_{\max }, \mathrm{C}_{\max }, \mathrm{t}_{1 / 2}, \mathrm{MRT}, \mathrm{CL}, \mathrm{Vd}$, and AUC, where $\mathrm{T}_{\max }$ and $\mathrm{C}_{\max }$ were actual measured values. All data were expressed as mean \pm standard deviation (SD).

\section{Results and Discussion}

\section{Method Validation and Improvement}

UPLC-MS/MS has the advantages of high sensitivity, strong specificity, short analysis time and good
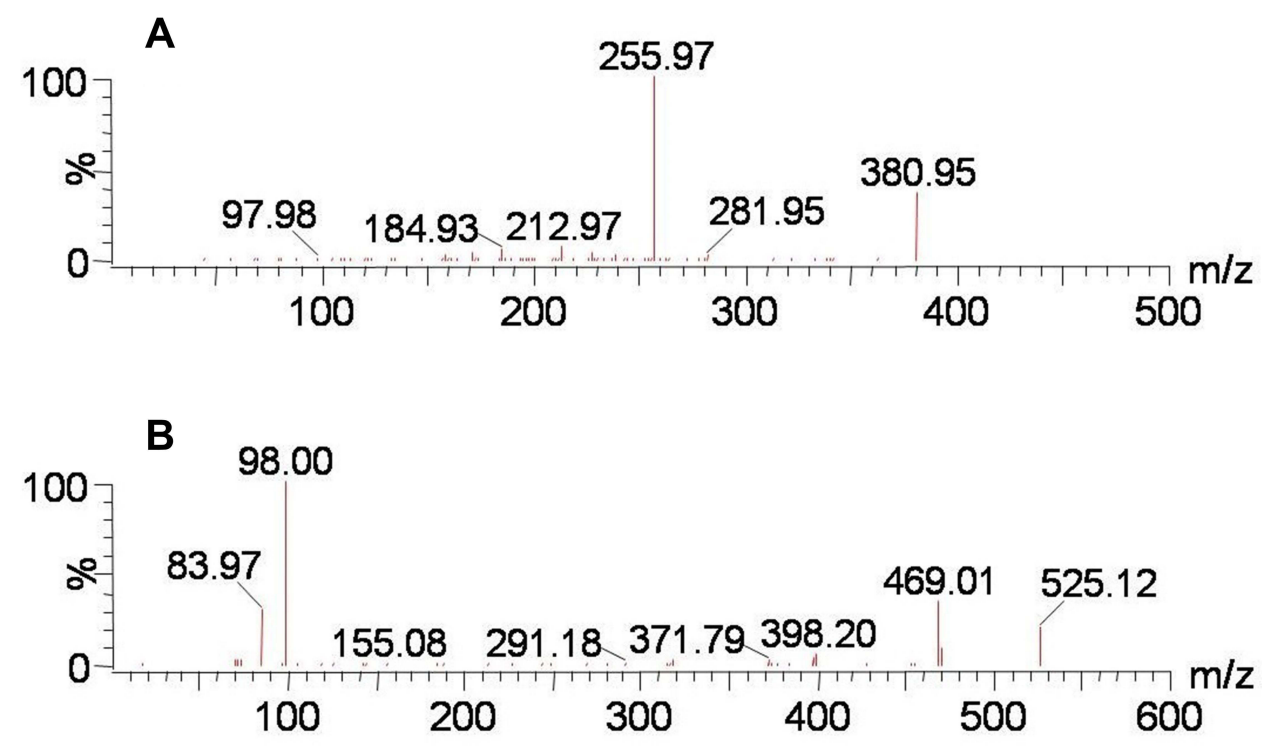

Figure 2 The Mass spectra of upadacitinib (A) and fedratinib (IS, B). 


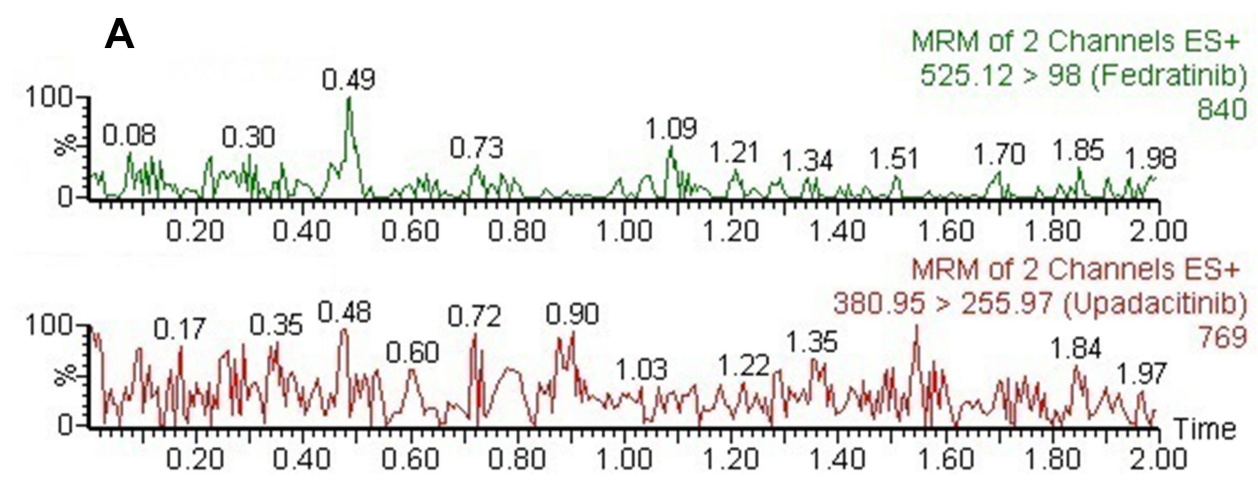

B
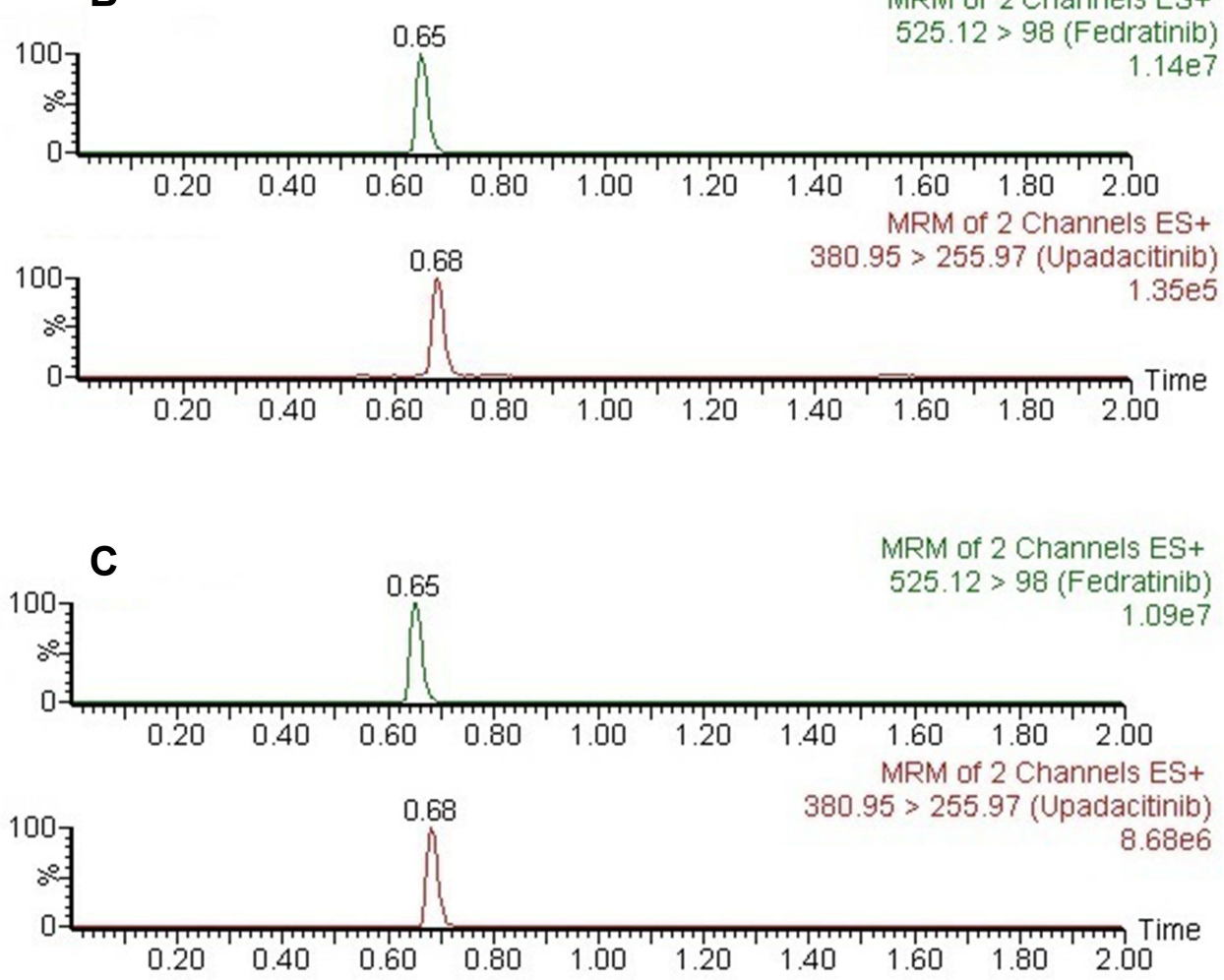

Figure 3 Representative chromatograms of upadacitinib and IS in beagle dog plasma. (A) A blank beagle dog plasma sample. (B) A beagle blank dog plasma sample spiked with upadacitinib I ng/mL (LLOQ) and IS. (C) A sample obtained from a beagle dog at $2 \mathrm{~h}$ after oral administration of $1.0 \mathrm{mg} / \mathrm{kg}$ upadacitinib.

reproducibility. Therefore, it is often used in the detection of drug concentration in biological samples, the study of pharmacokinetics and DDI. ${ }^{19,20}$

Fedratinib and upadacitinib were JAK inhibitors. Fedratinib was a selective JAK2 inhibitor for the treatment of adult patients with myelofibrosis (MF). Under the chromatographic and mass spectrometric conditions of this experiment, the peak shape of fedratinib and upadacitinib was good, the retention time was close and did not interfere with each other. At the same time, mass spectrometry detection had a good response. Therefore, fedratinib was selected as the internal standard of this experiment.
In this study, both ESI positive and negative were explored to discover the most sensitive ionization mode for upadacitinib and IS. It is demonstrated that the positive ion mode exhibits higher mass response than the negative ion mode. In positive full mass scan (as indicated in Figure 2), upadacitinib showed the most abundant protonated parent ion and daughter ion at $\mathrm{m} / \mathrm{z} 380.95$ and 255.97, respectively, and IS showed the most abundant parent ion and daughter ion at $\mathrm{m} / \mathrm{z} 525.12$ and 98.00, respectively. Therefore, the parent ion to the daughter ion of quantifier transitions were $m / z \quad 380.95 \rightarrow 255.97$ for upadacitinib, and $m / z 525.12 \rightarrow 98.00$ for IS, respectively. 
Table I The Precision and Accuracy of Upadacitinib in Beagle Dog Plasma $(n=6)$

\begin{tabular}{|l|c|c|c|c|c|c|}
\hline \multirow{2}{*}{ Added (ng/mL) } & \multicolumn{3}{|c|}{ Intra-Day } & \multicolumn{3}{c|}{ Inter-Day } \\
\cline { 2 - 7 } & Found (ng/mL) & RSD\% & RE\% & Found (ng/mL) & RSD\% & RE\% \\
\hline 1 & $0.97 \pm 0.10$ & 10.03 & -2.83 & $1.01 \pm 0.10$ & 9.68 & 0.95 \\
2.5 & $2.55 \pm 0.18$ & 6.96 & 1.84 & $2.56 \pm 0.16$ & 6.26 & 2.58 \\
50 & $48.10 \pm 2.87$ & 5.96 & -3.79 & $49.34 \pm 3.27$ & 6.62 & -1.37 \\
150 & $149.71 \pm 5.88$ & 3.93 & -0.19 & $153.26 \pm 7.77$ & 5.07 & 2.17 \\
\hline
\end{tabular}

Table 2 Recovery and Matrix Effect of Upadacitinib in Beagle Dog Plasma $(n=6)$

\begin{tabular}{|c|c|c|c|c|}
\hline \multirow[t]{2}{*}{ Added (ng/mL) } & \multicolumn{2}{|c|}{ Recovery (\%) } & \multicolumn{2}{|c|}{ Matrix Effect (\%) } \\
\hline & Mean \pm SD & RSD (\%) & Mean \pm SD & RSD (\%) \\
\hline 2.5 & $81.78 \pm 3.43$ & 4.20 & $101.09 \pm 3.26$ & 3.23 \\
\hline 50 & $82.69 \pm 3.21$ & 3.88 & $103.42 \pm 3.32$ & 3.21 \\
\hline 150 & $84.42 \pm 4.05$ & 4.80 & $98.48 \pm 4.77$ & 4.85 \\
\hline
\end{tabular}

Through the mass spectrometry standard sample injection, adjusting the mass spectrometry conditions, the characteristic parent ion and daughter ion to qualitative and quantitative were found out, and the specificity and accuracy were higher.

The mobile phase should meet the requirements of HPLC and LC/MS. Volatile salts should be added to the mobile phase as far as possible, surfactants and other non-volatile buffers should not be used as far as possible, such as phosphoric acid buffer. Phosphate and other non-volatile buffer salts will precipitate in the ion source and plug the capillary. In this experiment, formic acid, water and ammonium formate were first selected to investigate the influence of mobile phase on peak pattern and peak intensity. Finally, $0.1 \%$ formic acid and acetonitrile were used as mobile phase, which could increase the ionization degree of the sample, increase the signal intensity and improve the peak pattern.

In the field of pharmaceutical analysis, sample pretreatment and purification was an important step. Improving the pretreatment method could not only protect the detection instrument from pollution and prolong its service life but also reduce the detection matrix and improve the sensitivity and selectivity. In this study, through the exploration of a series of pretreatment methods, the ethyl acetate liquid-liquid extraction method was selected to pretreat the plasma samples, and treatment with $\mathrm{NaOH}$ during extraction process could alkalize plasma and significantly improve the extraction recovery rate of upadacitinib and IS. The influence of matrix interference on the determination was reduced.

\section{Selectivity}

Under the above experimental conditions, upadacitinib and IS were well separated completely, and the endogenous substances did not interfere with the detection. The representative chromatograms of a blank beagle dog plasma sample (A), a beagle blank dog plasma sample spiked with upadacitinib and IS (B), and a sample obtained from a beagle $\operatorname{dog}$ at $1.5 \mathrm{~h}$ after oral administration of $1.0 \mathrm{mg} / \mathrm{kg}$ upadacitinib (C) are shown in Figure 3. The retention times of upadacitinib and IS were 0.68 and $0.65 \mathrm{~min}$, respectively. The total running time for each sample was $2.0 \mathrm{~min}$.

\section{Calibration Curve and LLOQ}

At the concentration range of $1-200 \mathrm{ng} / \mathrm{mL}$ for upadacitinib, the typical regression equations of upadacitinib were $y=0.0754 * x+0.1276\left(r^{2}=0.9994\right)$, which exhibited an excellent linearity. The sensitivity of the method was detected by LLOQ and established as $1.0 \mathrm{ng} / \mathrm{mL}$, and the

Table 3 Stability Results of Upadacitinib in Plasma Under Different Conditions $(n=6)$

\begin{tabular}{|c|c|c|c|c|c|c|c|c|c|c|c|c|}
\hline \multirow{2}{*}{$\begin{array}{l}\text { Added (ng/ } \\
\mathrm{mL})\end{array}$} & \multicolumn{3}{|c|}{ Room Temperature, $12 \mathrm{~h}$} & \multicolumn{3}{|c|}{ Autosampler $4^{\circ} \mathrm{C}, 6 \mathrm{~h}$} & \multicolumn{3}{|c|}{ Three Freeze-Thaw } & \multicolumn{3}{|c|}{$-20^{\circ} \mathrm{C}, 4$ Weeks } \\
\hline & Mean \pm SD & $\begin{array}{r}\text { RSD } \\
\text { (\%) }\end{array}$ & $\begin{array}{l}\text { RE } \\
\text { (\%) }\end{array}$ & Mean \pm SD & $\begin{array}{c}\text { RSD } \\
\text { (\%) }\end{array}$ & $\begin{array}{l}\text { RE } \\
\text { (\%) }\end{array}$ & Mean \pm SD & $\begin{array}{c}\text { RSD } \\
\text { (\%) }\end{array}$ & $\begin{array}{l}\text { RE } \\
\text { (\%) }\end{array}$ & Mean \pm SD & $\begin{array}{c}\text { RSD } \\
(\%)\end{array}$ & $\begin{array}{l}\text { RE } \\
\text { (\%) }\end{array}$ \\
\hline 2.5 & $2.46 \pm 0.20$ & 8.21 & -1.73 & $2.48 \pm 0.22$ & 8.93 & -0.73 & $2.52 \pm 0.15$ & 6.15 & 0.87 & $2.42 \pm 0.14$ & 5.79 & -3.40 \\
\hline 50 & $51.14 \pm 2.63$ & 5.14 & 2.28 & $49.66 \pm 4.33$ & 8.73 & -0.69 & $50.11 \pm 4.02$ & 8.02 & 0.21 & $48.56 \pm 1.79$ & 3.68 & -2.87 \\
\hline 150 & $148.54 \pm 9.50$ & 6.39 & -0.98 & $151.80 \pm 6.19$ & 4.08 & 1.20 & $153.82 \pm 6.43$ & 4.18 & 2.55 & $149.63 \pm 7.42$ & 4.96 & -0.25 \\
\hline
\end{tabular}




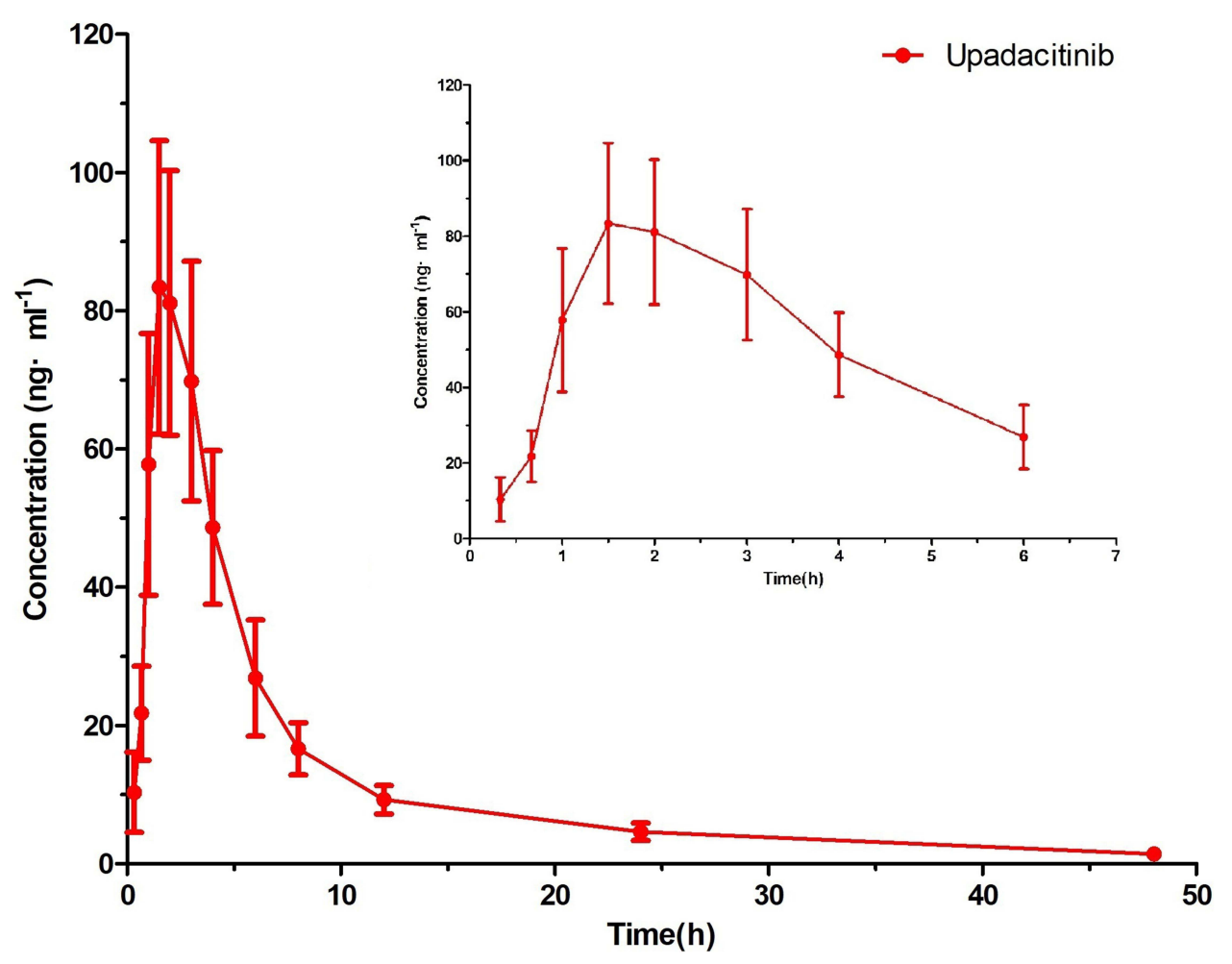

Figure 4 Mean plasma concentration-time curves of upadacitinib in beagle dogs after oral administration of upadacitinib at a single dose of $1.0 \mathrm{mg} / \mathrm{kg}(\mathrm{n}=6)$.

precision was below $10.03 \%$, whereas the accuracy ranged from $-2.83 \%$ to $0.95 \%$ (Table 1 ).

\section{Precision and Accuracy}

The results obtained for the intra- and inter-day precision and accuracy of upadacitinib are shown in Table 1. The precision ( $\%$ RSD) did not exceed 6.96\%. Accuracy (\% RE) was in the

Table 4 The Main Pharmacokinetic Parameters of Upadacitinib in Beagle Dog Plasma After Oral Administration of Upadacitinib at a Single Dose of $1.0 \mathrm{mg} / \mathrm{kg}(\mathrm{n}=6$, Mean \pm SD)

\begin{tabular}{|l|c|}
\hline Parameters & Upadacitinib \\
\hline $\mathrm{T}_{\max }(\mathrm{h})$ & $1.75 \pm 0.27$ \\
$\mathrm{C}_{\max }(\mathrm{ng} / \mathrm{mL})$ & $89.38 \pm 2 \mathrm{I} .3 \mathrm{I}$ \\
$\mathrm{t}_{\mathrm{I} / 2}(\mathrm{~h})$ & $\mathrm{II} .55 \pm \mathrm{I} .36$ \\
$\mathrm{MRT}_{0 \rightarrow \mathrm{t}}(\mathrm{h})$ & $9.27 \pm \mathrm{I} .08$ \\
$\mathrm{MRT}_{0 \rightarrow \infty}(\mathrm{h})$ & $\mathrm{II} .55 \pm \mathrm{I} .34$ \\
$\mathrm{CLz} / \mathrm{F}(\mathrm{L} / \mathrm{h} / \mathrm{kg})$ & $1.76 \pm 0.27$ \\
$\mathrm{Vz} / \mathrm{F}(\mathrm{L} / \mathrm{kg})$ & $29.05 \pm 4.32$ \\
$\mathrm{AUC} C_{0 \rightarrow \mathrm{t}}(\mathrm{ng} / \mathrm{mL} \cdot \mathrm{h})$ & $558.09 \pm 94.32$ \\
$\mathrm{AUC} C_{0 \rightarrow \infty}(\mathrm{ng} / \mathrm{mL} \cdot \mathrm{h})$ & $58 \mathrm{I} .85 \pm 98.67$ \\
\hline
\end{tabular}

Abbreviations: $T_{\max }$, time of peak concentration; $C_{\max }$, peak concentration; $t_{1 / 2}$, halflife; $M_{R T} T_{(0-t)}$, mean residence time of 0-t time; $M T_{(0-\infty)}$, mean residence time of 0 -infinity time; $\mathrm{CLz} / \mathrm{F}$, clearance rate; $\mathrm{Vz} / \mathrm{F}$, apparent volume of distribution; $\mathrm{AUC}_{(0-\mathrm{t})}$, area under curve of 0 -t time; $\mathrm{AUC}_{(0-\infty)}$, area under curve of 0 -infinity time. range from $-3.79 \%$ to $2.58 \%$ at low, medium and high concentrations and met the requirements of validation.

\section{Recovery and Matrix Effect}

At LQC, MQC and HQC three different concentration levels, the mean recovery of upadacitinib was within the range of $81.78 \% \sim 84.42 \%$ (Table 2), and the matrix effect values for upadacitinib were $98.48 \% \sim 103.42 \%$ (Table 2). Matrix effect did not affect the detection of samples.

\section{Stability}

All results of the stability are summarized in Table 3, and it is found that upadacitinib in beagle dog plasma is stable under the conditions described above.

\section{Pharmacokinetic Application}

The novel established UPLC-MS/MS method was successfully applied to a pharmacokinetic study after a single $1.0 \mathrm{mg} / \mathrm{kg}$ upadacitinib oral administration to six beagle dogs. The average plasma concentration-time curves of upadacitinib in beagle dogs are displayed in Figure 4, and the main pharmacokinetic parameters of upadacitinib are summarized in Table 4. 


\section{Conclusions}

A robust, quick and reliable UPLC-MS/MS assay was fully optimized and firstly developed to detect the plasma concentration of upadacitinib in beagle dogs. The optimized method had the advantage of short analysis time (2.0 $\mathrm{min}$ ), high recovery, good precision and strong specificity. After validation, the method had been successfully applied to the pharmacokinetic study of upadacitinib in beagle dogs. In future studies, this UPLC-MS/MS method would be applied to the study of DDIs, including the effects of Chinese herbal medicine or western medicine on the pharmacokinetics of upadacitinib.

\section{Author Contributions}

All authors made a significant contribution to the work reported, whether that is in the conception, study design, execution, acquisition of data, analysis and interpretation, or in all these areas; took part in drafting, revising or critically reviewing the article; gave final approval of the version to be published; have agreed on the journal to which the article has been submitted; and agree to be accountable for all aspects of the work.

\section{Disclosure}

The authors declare that they have no known competing financial interests or personal relationships that could have appeared to influence the work reported in this paper.

\section{References}

1. Smolen JS, Aletaha D, Barton A, et al. Rheumatoid arthritis. Nat Rev Dis Primers. 2018;4:18001. doi:10.1038/nrdp.2018.1

2. McInnes IB, Schett G. Pathogenetic insights from the treatment of rheumatoid arthritis. Lancet 2017;389:2328-2337. doi:10.1016/S01406736(17)31472-1

3. Parmentier JM, Voss J, Graff C, et al. In vitro and in vivo characterization of the JAK1 selectivity of upadacitinib (ABT-494). BMC Rheumatol. 2018;2(1):23. doi:10.1186/s41927-018-0031-x

4. Alunno A, Padjen I, Fanouriakis A, Boumpas DT. Pathogenic and therapeutic relevance of JAK/STAT signaling in systemic lupus erythematosus: integration of distinct inflammatory pathways and the prospect of their inhibition with an oral agent. Cells. 2019;8(8):898. doi: $10.3390 /$ cells 8080898

5. Kameda H, Fujii T, Nakajima A, et al. Japan college of rheumatology guideline for the use of methotrexate in patients with rheumatoid arthritis. Mod Rheumatol. 2019;29(1):31-40. doi:10.1080/ 14397595.2018.1472358

6. Smolen JS, Landewe RBM, Bijlsma JWJ, et al. EULAR recommendations for the management of rheumatoid arthritis with synthetic and biological disease-modifying antirheumatic drugs: 2019 update. Ann Rheum Dis. 2020;79(6):685-699. doi:10.1136/annrheumdis-2019216655
7. Norman P. Selective JAK inhibitors in development for rheumatoid arthritis. Expert Opin Investig Drugs. 2014;23(8):1067-1077. doi:10.1517/13543784.2014.918604

8. AbbVie. AbbVie receives FDA approval of RINVOQTM (upadacitinib), an oral JAK inhibitor for the treatment of moderate to severe rheumatoid arthritis; 2019. Available from: https://news.abbvie.com/ news/press-releases/abbvie-receives-fda-approval-rinvoqupadacitinib-an-oral-jak-inhibitor-for-treatment-moderate-to-severerheumatoid-arthritis.htm. Accessed November 28, 2019.

9. Conaghan PG, Mysler E, Tanaka Y, et al. Upadacitinib in rheumatoid arthritis: a benefit-risk assessment across a Phase III program. Drug Saf. 2021;44(5):515-530. doi:10.1007/s40264-020-01036-w

10. Mease PJ, Lertratanakul A, Papp KA, et al. Upadacitinib in patients with psoriatic arthritis and inadequate response to biologics: 56-week data from the randomized controlled Phase 3 SELECT-PsA 2 study. Rheumatol Ther. 2021;8(2):903-919. doi:10.1007/s40744-02100305-z

11. Mohamed MF, Camp HS, Jiang P, Padley RJ, Asatryan A, Othman AA. Pharmacokinetics, safety and tolerability of ABT-494, a novel selective JAK 1 inhibitor, in healthy volunteers and subjects with rheumatoid arthritis. Clin Pharmacokinet. 2016;55 (12):1547-1558. doi:10.1007/s40262-016-0419-y

12. Mohamed-Eslam F, Klünder MB, Othman AA. Clinical pharmacokinetics of Upadacitinib: review of data relevant to the rheumatoid arthritis indication. Clin Pharmacokinet. 2020;59(5):531-544. doi:10.1007/s40262-019-00855-0

13. Xuegu X, Luo S, Yang Q, et al. Development and validation of the quantitative determination of avapritinib in rat plasma by a bioanalytical method of UPLC-MS/MS. Arab J Chem. 2021;14 (6):103152. doi:10.1016/j.arabjc.2021.103152

14. Zhang Y, Zhu M, Xie S, Ye X, Xuegu X. Simultaneous determination of amiodarone, dronedarone, and their principal metabolites in SD rat plasma by UPLC-MS/MS and its application in pharmacokinetics. Arab J Chem. 2021;14(8):103300. doi:10.1016/j.arabjc.2021.103300

15. Mohamed MF, Zeng J, Marroum PJ, Song IH, Othman AA. Pharmacokinetics of Upadacitinib with the clinical regimens of the extended-release formulation utilized in rheumatoid arthritis Phase 3 trials. Clin Pharmacol Drug Dev. 2019;8(2):208-216. doi:10.1002/ cpdd. 462

16. Mohamed MF, Trueman S, Feng T, Anderson J, Marbury TC, Othman AA. Characterization of the effect of renal impairment on Upadacitinib pharmacokinetics. J Clin Pharmacol. 2019;59 (6):856-862. doi:10.1002/jcph.1375

17. US Food and Drug Administration. Guidance for industry: bioanalytical method validation. Rockville, MD, USA: US Department of Health and Human Services, US FDA, Center for Drug Evaluation and Research; 2018. Available from: https://www.fda.gov/regulator yinformation/search-fda-guidance-documents/bioanalyticalmethodva lidation-guidance-industry. Accessed August 10, 2018.

18. The technical guidelines for non clinical pharmacokinetics of drugs. Available from: http://www.nmpa.gov.cn/gsz05106/15.pdf. Accessed September 22, 2021

19. Hu J, Su X-J, Si H-L, et al. Simultaneous determination of Celecoxib, Dezocine and Dexmedetomidine in Beagle Plasma using UPLC-MS/ MS method and the application in pharmacokinetics. Drug Des Devel Ther. 2021;15:2529-2541. doi:10.2147/DDDT.S314562

20. Zhou C-J, Wang H-J, Zhou C-Y, et al. Establishment and verification of UPLC-MS/MS technique for pharmacokinetic drug-drug interactions of Selinexor with Posaconazole in rats. Drug Des Devel Ther. 2021;15:1561-1568. doi:10.2147/DDDT.S303928 


\section{Publish your work in this journal}

Drug Design, Development and Therapy is an international, peerreviewed open-access journal that spans the spectrum of drug design and development through to clinical applications. Clinical outcomes, patient safety, and programs for the development and effective, safe, and sustained use of medicines are a feature of the journal, which has also

been accepted for indexing on PubMed Central. The manuscript management system is completely online and includes a very quick and fair peer-review system, which is all easy to use. Visit http://www. dovepress.com/testimonials.php to read real quotes from published authors.

Submit your manuscript here: https://www.dovepress.com/drug-design-development-and-therapy-journal 